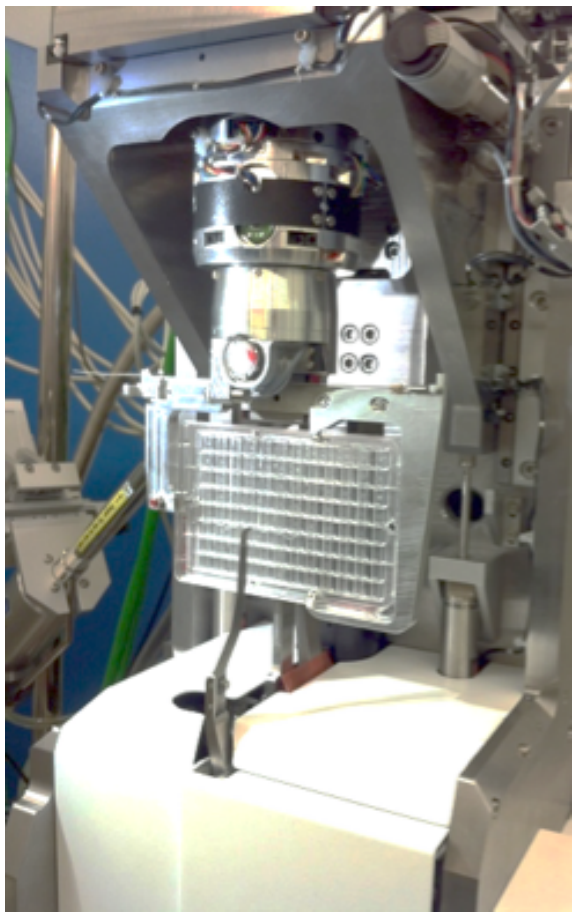

Figure 1. CrystalDirect ${ }^{\mathrm{TM}}$ crystallization plate mounted on the MD3 diffractometer for in situ data collection.

Keywords: in situ datacollection, LCP, serial crystallography

\section{MS2-O3 Lipidic Cubic Phase Injection for Serial X-ray Crystallography}

\author{
Daniel James ${ }^{1}$
}

1. Paul Scherrer Institut, Switzerland

email: daniel.james@psi.ch

High intensity $\mathrm{x}$-ray free electron laser (XFEL) pulses are destructive for biological samples. Serial femtosecond crystallography (SFX) experiments require sample to be replaced after each XFEL pulse, and have typically used liquid microjets to continuously renew sample. The high flow rate associated with these liquid microjets requires large volumes of sample, most of which runs to waste between shots. An injector designed to extrude a viscous gel-like material called lipidic cubic phase (LCP) was developed to address this problem. LCP, commonly used as a growth medium for membrane protein crystals, lends itself to low flow rate jetting and so reduces the amount of sample wasted significantly. Recently the injector has been used for serial crystallography experiments at synchrotrons, and has been shown to work with alternate viscous carrier fluids extending the usability of the injector.

Keywords: XFEL pulses, Lipidic Cubic Phase Injection 